\title{
Radiologic presentation of non-small cell lung cancer treated with anti-PD-1 therapy
}

\author{
Eun Young Kim ${ }^{1}$, Young Saing Kim², Inkeun Park ${ }^{2}$, Hee Kyung Ahn ${ }^{2}$, Hee Young Lee ${ }^{1}$ Jeong Ho Kim ${ }^{1}$ \\ ${ }^{1}$ Department of Radiology, Gil Medical Center, Gachon University College of Medicine, Incheon, Republic of Korea; ${ }^{2}$ Division of Medical \\ Oncology, Department of Internal Medicine, Gil Medical Center, Gachon University College of Medicine, Incheon, Republic of Korea \\ Correspondence to: Young Saing Kim, MD, PhD. Division of Medical Oncology, Department of Internal Medicine, Gil Medical Center, Gachon \\ University College of Medicine, 1198 Guwol-dong, Namdong-gu, Incheon 405-760, Republic of Korea. Email: zoomboom@hanmail.net. \\ Comment on: Hammer M, Bagley S, Aggarwal C, et al. Thoracic Imaging of Non-Small Cell Lung Cancer Treated With Anti-programmed Death \\ Receptor-1 Therapy. Curr Probl Diagn Radiol 2018. [Epub ahead of print]. \\ Katz SI, Hammer M, Bagley SJ, et al. Radiologic Pseudoprogression during Anti-PD-1 Therapy for Advanced Non-Small Cell Lung Cancer. J \\ Thorac Oncol 2018;13:978-86.
}

Submitted Aug 24, 2018. Accepted for publication Sep 23, 2018.

doi: $10.21037 /$ jtd.2018.09.129

View this article at: http://dx.doi.org/10.21037/jtd.2018.09.129

Tumors develop multiple mechanisms to evade attack from the immune system. Understanding these immune evasion mechanisms is important for developing new strategies in cancer treatment. Immune checkpoint inhibitors targeting the PD-1/PD-L1 axis yield remarkably durable responses in multiple solid cancer, such as melanoma, non-small cell lung cancer (NSCLC), renal cell carcinoma, and urothelial cancer (1-4). Currently, agents that have been FDA approved for clinical use are pembrolizumab and nivolumab (PD-1 inhibitors), atezolizumab, durvalumab, and avelumab (PDL1 inhibitors).

Immune checkpoint inhibitors induce inflammation towards the tumor, and make tumor cells to die. However, these agents can present great challenges to radiologists since their mode of action is distinct from conventional chemotherapeutic agents, which directly impede tumor cell growth. For example, immune checkpoint inhibitor treatment can lead to initial tumor enlargement with subsequent tumor regression, termed radiologic pseudoprogression, which can be misinterpreted as tumor progression on initial follow-up. Immune checkpoint inhibitors can also incite pneumonitis, sarcoid-like reactions, or other autoimmune reaction in normal tissues that can complicate image interpretation (5). Radiologically evident immune-related adverse events have been reported in up to approximately $30 \%$ of patients on immune checkpoint inhibitor therapy $(2,6)$.
In the articles entitled "Thoracic Imaging of NonSmall Cell Lung Cancer Treated with Anti-programmed Death Receptor-1 Therapy" (7) and "Radiologic Pseudoprogression during Anti-PD-1 Therapy for Advanced Non-Small Cell Lung Cancer" (8) recently published in Current Problems in Diagnostic Radiology and Fournal of Thoracic Oncology, respectively, by Hammer $\mathrm{M}$, Katz S and colleagues. The authors retrospectively reviewed consecutive patients with NSCLC receiving antiPD-1 therapy at their institution between 2013 and 2016, and they evaluate the incidence and imaging findings of radiologic pseudoprogression and anti-PD-1 treatmentinduced pneumonitis. Radiologic pseudoprogression was determined when tumors showed enlargement or new lesions were detected at first follow-up CT and subsequent decrease in tumor burden at second followup CT on sustained anti-PD-1 therapy. CT findings were also evaluated in patients with clinically evident antiPD-1 treatment-related pneumonitis (shortness of breath, hypoxia, or cough developing following the initiation of anti-PD-1 treatment, with no clinical evidence of infection, and symptoms improving with steroids or on termination of anti-PD-1 therapy).

A total of 166 adult patients with NSCLC (mean age: 73 years, range: 34 to 88 years) who were treated with an anti-PD-1 drug [either nivolumab (80\%) or pembrolizumab $(20 \%)]$ were available for the analysis; most common 
histology was adenocarcinoma of the lung (73\%). According to RECIST 1.1 , the overall response rate was $22 \%$ [complete response $(n=4)$ and partial response $(n=32)]$, and $53 \%$ of patients $(\mathrm{n}=88)$ demonstrated progressive disease (PD) at first follow-up CT. Radiologic pseudoprogression was clinically suspected in 15 patients ( $9 \%$ of the cohort) because of clinical improvement despite imaging evidence of an increase in tumor burden; these patients sustained the immune checkpoint inhibitor therapy. Most of these patients confirmed as true progression on subsequent follow up. Only three patients ( $2 \%$ of the cohort) confirmed as radiologic pseudoprogression, with decrease in tumor burden on subsequent follow-up CT after 4-6 months of therapy. At first follow-up CT, 2 of the 3 cases of radiologic pseudoprogression manifested as enlargement of the primary tumor. And one patient demonstrated a decrease in size of the primary tumor with the development of new pulmonary lesions. One additional patient with stable disease according to RECIST 1.1 at the first follow-up CT manifested a subsequent $18 \%$ reduction in tumor burden at second follow-up, which suggest the pattern of radiologic pseudoprogression.

Among a total of 5 patients (3\%) who developed clinically significant anti-PD-1 therapy-induced pneumonitis, four patients had changes on CT obtained at 2-11 weeks of therapy (mean 8 weeks). In two patients, pneumonitis developed as peribronchial, peripheral, or subpleural patchy ground glass opacities. In two patients, pneumonitis manifested as consolidation in the region of concurrent or recent prior radiotherapy. In the literature, imaging findings of anti-PD-1 treatment-related pneumonitis include patterns of non-specific interstitial pneumonia (NSIP), organizing pneumonia, and diffuse alveolar damage, which are usually observed in the first 3 months of therapy, although some reports of delayed emergence of pneumonitis also have been reported (5,9-12).

In the radiologic pseudoprogression have been observed in clinical trials with the incidence of $0-5 \%(13-16)$; the transient enlargement of baseline tumor burden and new lesions at first follow-up are associated with immunerelated response including development of edema and infiltrates of immune cells. Since the finding of radiologic pseudoprogression would have been classified prematurely as PD by conventional tumor response criteria (i.e., historic WHO or RECIST criteria), immune-related response criteria such as unidimensional immune-related response criteria (iRRC) and iRECIST have been developed $(17,18)$. The unidimensional iRRC and iRECIST have an improved capacity to capture radiologic pseudoprogression than RECIST 1.1. In this study cohort, no patients were confirmed as having PD using iRRC and iRECIST at first follow-up CT. However, the assessment is delayed since these immune-related response criteria require suspected $\mathrm{PD}$ to be confirmed by subsequent follow-up imaging at least 1 month later.

In summary, radiologic pseudoprogression occurred infrequently (2\%) in NSCLC patients receiving anti-PD-1 therapy. Radiologically evident anti-PD-1 therapy-induced pneumonitis was observed in $3 \%$ of this cohort, and the radiologic findings were either patchy ground glass opacities or consolidation in the region of concurrent or recent prior radiation therapy.

\section{Acknowledgements}

None.

\section{Footnote}

Conflicts of Interest: The authors have no conflicts of interest to declare.

\section{References}

1. Aguiar PN Jr, Santoro IL, Tadokoro H, et al. A pooled analysis of nivolumab for the treatment of advanced non-small-cell lung cancer and the role of PD-L1 as a predictive biomarker. Immunotherapy 2016;8:1011-9.

2. Chiou VL, Burotto M. Pseudoprogression and ImmuneRelated Response in Solid Tumors. J Clin Oncol 2015;33:3541-3.

3. Hakenberg OW. Nivolumab for the treatment of bladder cancer. Expert Opin Biol Ther 2017;17:1309-15.

4. Schadendorf D, Wolchok JD, Hodi FS, et al. Efficacy and Safety Outcomes in Patients With Advanced Melanoma Who Discontinued Treatment With Nivolumab and Ipilimumab Because of Adverse Events: A Pooled Analysis of Randomized Phase II and III Trials. J Clin Oncol 2017;35:3807-14.

5. Nishino M, Sholl LM, Hodi FS, et al. Anti-PD-1-Related Pneumonitis during Cancer Immunotherapy. N Engl J Med 2015;373:288-90.

6. Tirumani SH, Ramaiya NH, Keraliya A, et al. Radiographic Profiling of Immune-Related Adverse Events in Advanced Melanoma Patients Treated with Ipilimumab. Cancer Immunol Res 2015;3:1185-92. 
7. Hammer M, Bagley S, Aggarwal C, et al. Thoracic Imaging of Non-Small Cell Lung Cancer Treated With Anti-programmed Death Receptor-1 Therapy. Curr Probl Diagn Radiol 2018. [Epub ahead of print].

8. Katz SI, Hammer M, Bagley SJ, et al. Radiologic Pseudoprogression during Anti-PD-1 Therapy for Advanced Non-Small Cell Lung Cancer. J Thorac Oncol 2018;13:978-86.

9. Gounant V, Brosseau S, Naltet C, et al. Nivolumabinduced organizing pneumonitis in a patient with lung sarcomatoid carcinoma. Lung Cancer 2016;99:162-5.

10. Kato T, Masuda N, Nakanishi Y, et al. Nivolumab-induced interstitial lung disease analysis of two phase II studies patients with recurrent or advanced non-small-cell lung cancer. Lung Cancer 2017;104:111-8.

11. Nishino M, Chambers ES, Chong CR, et al. Anti-PD-1 Inhibitor-Related Pneumonitis in Non-Small Cell Lung Cancer. Cancer Immunol Res 2016;4:289-93.

12. Tada K, Kurihara Y, Myojo T, et al. Case report of nivolumab-related pneumonitis. Immunotherapy 2017;9:313-8

13. Somarouthu B, Lee SI, Urban T, et al. Immune-related

Cite this article as: Kim EY, Kim YS, Park I, Ahn HK, Lee HY, Kim JH. Radiologic presentation of non-small cell lung cancer treated with anti-PD-1 therapy. J Thorac Dis 2018;10(Suppl 33):S3930-S3932. doi: 10.21037/jtd.2018.09.129 tumour response assessment criteria: a comprehensive review. Br J Radiol 2018;91:20170457.

14. Borghaei H, Paz-Ares L, Horn L, et al. Nivolumab versus Docetaxel in Advanced Nonsquamous Non-Small-Cell Lung Cancer. N Engl J Med 2015;373:1627-39.

15. Gettinger SN, Horn L, Gandhi L, et al. Overall Survival and Long-Term Safety of Nivolumab (Anti-Programmed Death 1 Antibody, BMS-936558, ONO-4538) in Patients With Previously Treated Advanced Non-Small-Cell Lung Cancer. J Clin Oncol 2015;33:2004-12.

16. Nishino M, Ramaiya NH, Chambers ES, et al. Immunerelated response assessment during $\mathrm{PD}-1$ inhibitor therapy in advanced non-small-cell lung cancer patients. J Immunother Cancer 2016;4:84.

17. Nishino M, Giobbie-Hurder A, Gargano M, et al. Developing a common language for tumor response to immunotherapy: immune-related response criteria using unidimensional measurements. Clin Cancer Res 2013;19:3936-43.

18. Seymour L, Bogaerts J, Perrone A, et al. iRECIST: guidelines for response criteria for use in trials testing immunotherapeutics. Lancet Oncol 2017;18:e143-52. 